\title{
A REDUCTION OF THE POINCARE CONJECTURE TO OTHER CONJECTURES
}

\author{
BY C. D. PAPAKYRIAKOPOULOS ${ }^{1}$ \\ Communicated by Edwin Moise, March 4, 1962
}

In the present note we explain briefly our method and state the theorems without proofs. These things will be explained in detail in a subsequent paper, which will be published in another journal. Our problem is the following.

PoinCaré CONJeCtURe. Any simply-connected closed ${ }^{2}$ 3-manifold, i.e., any homotopy 3-sphere, is a 3-sphere.

The generalized Poincaré conjecture has been proved for dimensions $\geqq 5$ by S. Smale [9], J. R. Stallings [10], E. C. Zeeman [12], and A. H. Wallace [11]. For dimension 4 nothing is known, to the best knowledge of this author.

1. The diagram. Let $M=T \cup T^{\prime}$, where $M$ is an orientable closed 3 -manifold, $T$ and $T^{\prime}$ are handlebodies ${ }^{8}$ of the same genus $p(\geqq 0)$, and $N=$ bd $T=$ bd $T^{\prime}=T \cap T^{\prime}$ is an orientable closed $^{2}$ surface of genus $p$. We then say that $M=T \cup T^{\prime}$ is a Heegaard splitting of genus $p$ of $M$. Let $A_{i}, B_{i}, i=1, \cdots, p$, be a fundamental system of $N$ based at $o$, i.e., the $A, B$ 's are simple loops on $N$ based at $o$, having only the point $o$ in common, and such that if we cut $N$ along the $A, B$ 's we obtain a 2-cell with boundary

$$
\prod_{i=1}^{p} A_{i} B_{i} A_{i}^{-1} B_{i}^{-1}
$$

From now on, unless otherwise stated, we suppose that $p \geqq 2$. Let $A_{i}, B_{i}$ be a system as above, such that $A_{i} \simeq 0$ in $T$. Let $\phi: N \rightarrow N$ be an homeomorphism, such that $\phi\left(A_{i}\right) \simeq 0$ in $T^{\prime}$. Let $\psi: F \rightarrow F$ be the automorphism induced by $\phi$, where

$$
\pi_{1}(N, o) \approx F=\left(a_{1}, b_{1}, \cdots, a_{p}, b_{p}: \prod_{i=1}^{p}\left[a_{i}, b_{i}\right]\right), \quad p \geqq 2 .
$$

1 The author is an Alfred P. Sloan Foundation Research Fellow.

${ }^{2}$ Closed means compact without boundary.

${ }^{3}$ Henkelkörper vom Geschlechte $p[8$, p. 219, §63]. In my previous papers [5, p. 281; 7 , p. 325, No. 10], I called these solid tori of genus $p$. However I now adopt the term handlebody of genus $p$ since it has been used in the literature.

$\iota \simeq$ means homotopic to, $\sim$ means homologous to. 
Let finally $A=\left\langle a_{1}, \cdots, a_{p}\right\rangle, \tilde{\Delta}=A \cap \psi(A), \hat{\Delta}=\langle A, \psi(A)\rangle$.

TheOREM (1.1), $\pi_{1}(M, o) \approx F / \hat{\Delta}$. Therefore $M$ is simply-connected if and only if $\hat{\Delta}=F$.

This is proved directly from the above.

Lemma (1.2). The groups $A / \widetilde{\Delta}, \psi(A) / \widetilde{\Delta}, \hat{\Delta} / \tilde{\Delta}$, and $F / \tilde{\Delta}$ have no elements of finite order. $\hat{\Delta}, \tilde{\Delta}$ are normal subgroups of $F$, such that $\tilde{\Delta} \subset \hat{\Delta}$ and $1 \neq \tilde{\Delta} \neq F$.

That $\widetilde{\Delta} \neq 1$ is proved using some properties of $F$, which are obtained from the Poincare model of the hyperbolic plane, as this is used in the Nielsen theory, see for example $[3 ; 4]$. The other properties are proved using group theory. From $\tilde{\Delta} \neq 1$ follows.

THEOREM (1.3). There exists on $N$ a loop $L$, which is $\Varangle 0$ on $N$, but it is $\simeq 0$ both in $T$ and $T^{\prime}$. However $L$ is not simple, generally.

Let $\tau: \widetilde{T} \rightarrow T$ or $\tau^{\prime}: \widetilde{T}^{\prime} \rightarrow T^{\prime}$ be the universal coverings of $T$ or $T^{\prime}$ respectively, and let $\tilde{N}=$ bd $\tilde{T}, \tilde{N}^{\prime}=\mathrm{bd} \tilde{T}^{\prime}$. The following is proved using geometric techniques.

Lemma (1.4). $\tilde{N}$ and $\tilde{N}^{\prime}$ are planar ${ }^{6}$ surfaces.

Let us consider the diagram

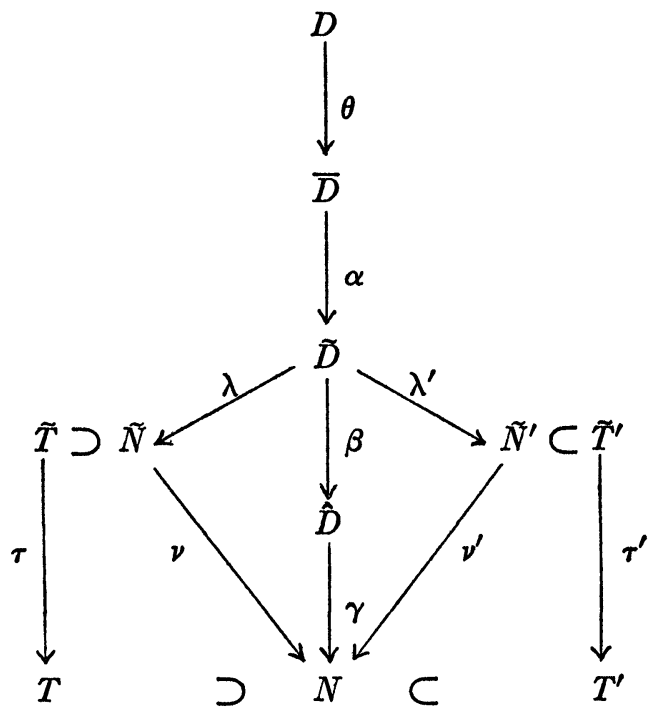

$\langle\langle\cdots\rangle$ means the smallest normal subgroup of $F$, containing the elements $\cdots$.

- Planar surface is one that is homeomorphic to an open connected subset of a 2-sphere. 
where $\gamma \beta: \widetilde{D} \rightarrow N$ and $\gamma: \hat{D} \rightarrow N$ are the regular coverings corresponding to the normal subgroups $\tilde{\Delta}$ and $\hat{\Delta}$ of $F$ respectively. Moreover $\gamma \beta \alpha: \bar{D} \rightarrow N$ is the regular covering corresponding to a normal subgroup $\bar{\Delta}$ of $F$, where $1 \neq \bar{\Delta} \subset \bar{\Delta}$ and which will be defined more precisely later on. Finally $D$ is the Poincare model of the hyperbolic plane.

The problems related with $\bar{\Delta}$ and $\bar{D}$ form the main difficulties of our method. We emphasize that, $\bar{\Delta}$ may be $=$ or $\neq \tilde{\Delta}$.

2. Geodesoids. Let us consider the sequence

$$
D \stackrel{\zeta}{\rightarrow} \dot{D} \stackrel{\eta}{\rightarrow} N
$$

where $\eta: \dot{D} \rightarrow N$ is the regular covering corresponding to the (not necessarily proper) normal subgroup $\dot{\Delta} \neq 1$ of $F$, and $\zeta: D \rightarrow \dot{D}$ is the universal covering of $\dot{D}$.

An oriented closed curve $\dot{G}$ on $\dot{D}$, which has at most "double" points and which is $\not 00$ on $\dot{D}$, is called a geodesoid if $\zeta^{-1}(\dot{G})$ consists of simple arcs, such that any two different ones have at most one point in common, but no end point in common.

Lemma (2.1). Let $\dot{G}$ be a geodesoid on $\dot{D}$ and let $\dot{J}$ be an oriented closed curve on $\dot{D}$, which has at most "double" points, such that $\dot{J} \simeq \dot{G}$ on $\dot{D}$. Then the number of double points of $\dot{G}$ is $\leqq$ the number of double points of $\dot{J}$, i.e., $c(\dot{G}) \leqq c(\dot{J})$, and if equality holds then $\dot{J}$ is a geodesoid.

This is proved using the fact that $D$ is the Poincare model of the hyperbolic plane, and using the properties of the geodesics on $D$ corresponding to the elements of $F$. The geodesoids have the main properties of the geodesics, but also "flexibility".

Let us now consider the sequence

$$
D \stackrel{\theta}{\rightarrow} \bar{D} \stackrel{\xi}{\rightarrow} \dot{D} \stackrel{\eta}{\rightarrow} N
$$

where $\eta: \dot{D} \rightarrow N$ and $\eta \xi: \bar{D} \rightarrow N$ are regular coverings, corresponding to the normal subgroups $\dot{\Delta}$ and $\bar{\Delta}$ of $F$, and having the following properties (2.2)-(2.4). For the purposes of this No. 2 it is not needed to suppose that $\bar{\Delta} \subset \tilde{\Delta}$ as was supposed in No. 1 .

(2.2) $\bar{\Delta}$ is a nontrivial proper subgroup of $\dot{\Delta}$, but $\dot{\Delta}$ may not be a proper subgroup of $F$.

(2.3) $\dot{\Delta} / \bar{\Delta}$ has no elements of finite order.

(2.4) $\bar{D}$ is planar. ${ }^{6}$

Let $(\dot{D}, \bar{D})$ be the set of all geodesoids of $\dot{D}$, which are covered just once by simple geodesoids of $\bar{D}$. Using the geodesics on $\bar{D}$, we prove 
that $(\dot{D}, \bar{D}) \neq \varnothing$. For any $\dot{G} \in(\dot{D}, \bar{D})$ we define the notions of complexity $c(\dot{G})$ and complementary complexity $C(\dot{G})$ of $\dot{G}$, just as we did in [5, pp. 282-283] for circuits. We say that $\dot{G} \in(\dot{D}, \bar{D})$ is an extremal element of this set, if there is no $\dot{J} \in(\dot{D}, \bar{D})$ such that, either $c(\dot{J})$ $<c(\dot{G})$, or $c(\dot{J})=c(\dot{G})$ and $C(\dot{J})<C(\dot{G})$.

ThEOREM (2.5). Under the conditions (2.2)-(2.4), any extremal element of $(\dot{D}, \bar{D})$ is simple.

The proof of this is very complicated. It makes use of an operation by means of which I proved the loop theorem [5, p. 281, Theorem (1) ]. However the short cut due to J. H. C. Whitehead, and explained in [5, pp. 285-293], does not work here, while the longer operation (unpublished) works almost word for word.

3. Necessary and sufficient conditions for the decomposition of 3-manifolds. We now return to the notations and conventions of No. 1. The following is the key theorem of this note.

THEOREM (3.1). There exists a simple loop $L$ on $N$, such that $L \not \neq 0$ on $N$, and $L \simeq 0$ both in $T$ and $T^{\prime}$ if, and only if, there exists a normal subgroup $\bar{\Delta}$ of $F$, such that $1 \neq \bar{\Delta} \subset \widetilde{\Delta}, F / \bar{\Delta}$ has no element of finite order, and $\bar{D}$ is planar.

Proof. The sufficiency of the conditions is proved the following way. Consider the diagram of No. 1 . Here we use the fact that $\bar{\Delta} \subset \tilde{\Delta}$. Suppose now that $\dot{\Delta}=F$, whence $\dot{D}=N$. Let $L$ be an extremal element of $(N, \bar{D})$. By Theorem (2.5), $L$ is a simple loop on $N$. Let $\bar{L}$ be a loop on $\bar{D}$ covering $L$. Then

$$
\begin{aligned}
L=\gamma \beta \alpha(\bar{L}) & =\nu \lambda \alpha(\bar{L}) \simeq 0 \text { in } T, \\
& =\nu^{\prime} \lambda^{\prime} \alpha(\bar{L}) \simeq 0 \text { in } T^{\prime}
\end{aligned}
$$

because $\lambda \alpha(\bar{L}) \simeq 0$ in $\widetilde{T}, \lambda^{\prime} \alpha(\bar{L}) \simeq 0$ in $\widetilde{T}^{\prime}$, because $\widetilde{T}$ and $\widetilde{T}^{\prime}$ are universal coverings of $T$ and $T^{\prime}$ respectively. Moreover $L \not \chi 0$ on $N$, because $L$ is a geodesoid on $N$. The proof of the necessity of the conditions does not make use of the notations introduced above, but it is involved.

The above theorem (3.1) helps us explain the cause of the difficulty of the classification problem for orientable closed 3-manifolds.

(3.2) The complexity of the classification problem for orientable closed 3-manifolds is due to the fact that: there exist covering spaces of planar ${ }^{6}$ surfaces which are not planar. 
Let us suppose that: any regular covering space of any planar surface is planar. Then $\tilde{D}$ is planar, because so is $\tilde{N}$, see Lemma (1.4). By Lemma (1.2), $F / \widetilde{\Delta}$ has no elements of finite order and $1 \neq \tilde{\Delta}$. Thus by Theorem (3.1), Dehn's Lemma [6], and induction on $p$ would follow that any orientable closed 3-manifold would be the composition of a 3-sphere with handles ${ }^{7}$ and a finite number of lens spaces. ${ }^{8}$ Hence the cause of our difficulties is the following.

(3.3) Generally, $\widetilde{D}$ is not planar.

The following remarks of R. H. Fox indicate that Proposition (3.2) is not as awkward as it may look: let $\widetilde{S} \rightarrow S$ be a finite sheeted branched covering over the 2 -sphere $S$. Deleting the branch points of $\widetilde{S}$ and their projections on $S$, we obtain an (unbranched) covering $\tilde{S}^{\prime} \rightarrow S^{\prime}$. It is well known that among all possible $\tilde{S}$ there are surfaces of genus $>0$. Therefore among all possible $\tilde{S}^{\prime}$ there are nonplanar surfaces, while all possible $S^{\prime}$ are planar.

4. Homotopy spheres. Let us now suppose that $M$ is a homology 3 -sphere, and let $T$ be a handlebody of genus $p \geqq 1$, semi-linearly imbedded in $M$, and let $N=$ bd $T$. The following holds.

THEOREM (4.1.). If $M$ is a homology 3-sphere, then there exists a system of oriented simple closed curves $X_{j}, Y_{j}, j=1, \cdots, p(\geqq 1)$, on $N$ such that: (i) the $X$-or $Y$-curves are disjoint; (ii) $X_{j}$ meets only $Y_{j}$ and only at one point; (iii) if we cut $N$ along $X_{j}$ and $Y_{j}$ we obtain a 2-sphere with $p$ holes, having boundaries $X_{j} Y_{j} X_{j}^{-1} Y_{j}^{-1}$; (iv) ${ }^{4} X_{j} \simeq 0$ in $T$, and $Y_{j} \sim 0$ in $\mathrm{cl}(M-T)$.

The case $p=1$ has been proved by M. Dehn [1]. The case $p \geqq 2$ is proved by induction and the proof is rather geometric. We call $X_{j}, Y_{j}, j=1, \cdots, p$, a meridian-longitude system of $T \subset M$ and Theorem (4.1) is called the "meridian-longitude theorem".

Let us now suppose that $M$ is an homotopy 3 -sphere and let $M=T \cup T^{\prime}$ be a Heegaard splitting of it. By Theorem (4.1), there is a meridian-longitude system $X_{j}, Y_{j}$ or $X_{j}^{\prime}, Y_{j}^{\prime}$ of $T \subset M$ or $T^{\prime} \subset M$ respectively. The Theorem (1.1), in conjunction with an algebraic lemma, enable us to perform two elementarytransformations of the system $X_{j}, Y_{j}$, by means of which, we obtain the following.

Theorem (4.2). If $M$ is a homotopy 3-sphere, and if $X_{j}^{\prime}, Y_{j}^{\prime}$, $j=1, \cdots, p(\geqq 1)$, is a meridian-longitude system of $T^{\prime} \subset M$, then

${ }^{7}$ See $[6$, p. $23, \S 8]$.

8 This is not true, because there are orientable closed 3-manifolds with finite not cyclic fundamental group, see [8, pp. 216-218]. 
there exists a meridian-longitude system $X_{j}, Y_{j}$ of $T \subset M$, such that $Y_{j} \sim X_{j}^{\prime}$ on $N$.

By an isotopic deformation of each one of the pairs $X_{j}, Y_{j}$ and $X_{j}^{\prime}, Y_{j}^{\prime}$ on $N$, we obtain new systems $A_{j}, B_{j}$ and $A_{j}^{\prime}, B_{j}^{\prime}$ based at 0 , such that both $A_{j}, B_{j}$ and $A_{j}^{\prime}, B_{j}^{\prime}, j=1, \cdots, p$, are fundamental systems of $N$. Let us denote by $a_{j}, b_{j}, a_{j}^{\prime}$ the elements of $\pi_{1}(N, 0)$ corresponding to $A_{j}, B_{j}, A_{j}^{\prime}$ respectively. By Theorem (4.2), $a_{j}^{\prime}=b_{j} \tau_{j}$, where $\tau_{j} \in[\Phi, \Phi]$, and $\Phi$ is the free group freely generated by $a_{1}, b_{1}, \cdots, a_{p}, b_{p}$.

5. Two conjectures. Let us now consider the special case where one of the $\tau$ 's, say $\tau_{1}$, is 1 . Let us define $\mathrm{e}^{5} \bar{\Delta}=\left\langle\left[a_{1}, a_{1}^{\prime}\right]\right\rangle$. Then using the Poincaré model of the hyperbolic plane, we prove that

$$
\begin{aligned}
1 & \neq \bar{\Delta} \subset\left\langle a_{1}, \cdots, a_{p}\right\rangle \cap \psi\left(\left\langle a_{1}, \cdots, a_{p}\right\rangle\right) \\
F / \bar{\Delta} & \approx\left(a_{1}, b_{1}, \cdots, a_{p}, b_{p}: \prod_{i=1}^{p}\left[a_{i}, b_{i}\right],\left[a_{1}, b_{1}\right]\right) \\
& \approx\left(a_{1}, b_{1}:\left[a_{1}, b_{1}\right]\right) *\left(a_{2}, b_{2}, \cdots, a_{p}, b_{p}: \prod_{i=2}^{p}\left[a_{i}, b_{i}\right]\right)
\end{aligned}
$$$$
p \geqq 2 \text {. }
$$

and therefore it has no elements of finite order. Finally the regular covering $\bar{D}$ of $N$ corresponding to $\bar{\Delta}$ is planar, because there exists a simple loop homotopic on $N$ to the loop representing $\left[a_{1}, b_{1}\right]$. Thus by Theorem (3.1), there is on $N$ a simple loop $L$, which is $\not 0$ on $N$, but $\simeq 0$ in both the handlebodies $T$ and $T^{\prime}$.

The above suggests that something similar may happen in the general case, where all $\tau$ 's are $\neq 1$. This leads us to the formulation of the following two conjectures.

(5.1) For some subset $(m, \cdots, n)$ of $(1, \cdots, p)$ the group $\left(a_{1}, b_{1}, \cdots, a_{p}, b_{p}: \prod_{i=1}^{p}\left[a_{i}, b_{i}\right],\left[a_{m}, b_{m} \tau_{m}\right], \cdots,\left[a_{n}, b_{n} \tau_{n}\right]\right)$ has no elements of finite order, where $\tau_{m}, \cdots, \tau_{n}$ are $\in[\Phi, \Phi]$.

(5.2) The regular covering space $\bar{D}_{\tau}$ of $N$, corresponding to $\bar{\Delta}_{\tau}$ $=\left\langle\left[a_{m}, b_{m} \tau_{m}\right], \cdots,\left[a_{n}, b_{n} \tau_{n}\right]\right\rangle$, is planar. ${ }^{6}$

In our problem $b_{m} \tau_{m}=a_{m}^{\prime}, \cdots, b_{n} \tau_{n}=a_{n}^{\prime}$ are represented by simple loops on $N$. This condition may turn out to be very important for the validity of the conjectures (5.1) and (5.2).

We observe that, the group of covering translations $F / \bar{\Delta}_{\tau}$ of the regular covering $\bar{D}_{\tau} \rightarrow N$ is the one appearing in (5.1).

Actually the conjectures (5.1) and (5.2) imply the Poincaré conjecture. However they seem to be very hard problems indeed.

As far as the conjecture (5.1) is concerned see A. Karrass, W. Mag- 
nus and D. Solitar [2]. We intend to handle the conjectures (5.1) and (5.2) in subsequent notes.

The author of the present article would like to express his gratitude to Professor R. H. Fox, for many and valuable discussions.

\section{BIBLIOGRAPHY}

1. M. Dehn, Über die Topologie des dreidimensionalen Raumes, Math. Ann. 69 (1910), 137-168.

2. A. Karrass, W. Magnus and D. Solitar, Elements of finite order in groups with a single defining relation, Comm. Pure Appl. Math. 13 (1960), 57-66.

3. J. Nielsen, Untersuchungen zur Topologie der geschlossenen zweiseitigen Flächen. I, Acta Math. 50 (1927), 189-358.

4. - Über Gruppen linearer Transformationen, Mitteil. Math. Gesellschaft Hamburg 8 (1940), 82-104.

5. C. D. Papakyriakopoulos, On solid tori, Proc. London Math. Soc. (3) 7 (1957), 281-299.

6. - On Dehn's lemma and the asphericity of knots, Ann. of Math. (2) 66 (1957), 1-26.

7. - Some problems on 3-dimensional manifolds, Bull. Amer. Math. Soc. 64 (1958), 317-335.

8. H. Seifert und W. Threlfall, Lehrbuch der Topologie, Teubner, Leipzig, 1934.

9. S. Smale, Generalized Poincarê's conjecture in dimensions greater than four, Ann. of Math. (2) 74 (1961), 391-406.

10. J. R. Stallings, Polyhedral homotopy-spheres, Bull. Amer. Math. Soc. 66 (1960), 485-488.

11. A. H. Wallace, Modifications and cobounding manifolds. II, J. Math. Mech. 10 (1961), 773-809.

12. E. C. Zeeman, The generalized Poincaré conjecture, Bull. Amer. Math. Soc. 67 (1961), 270.

\section{Princeton UnIVERSITY}

